\title{
AN EXTERNAL SHOCK ORIGIN OF GRB 141028A
}

\section{J. Michael*}

Max-Planck-Institut fãijr extraterrestrische Physik

E-mail: jburgess@mpe.mpg.de

\begin{abstract}
The prompt emission of the long, smooth, and single-pulsed gamma-ray burst, GRB 141028A, is analyzed under the guise of an external shock model. First, we fit the Îş-ray spectrum with a two-component photon model, namely, synchrotron+blackbody, and then fit the recovered evolution of the synchrotron $v F_{v}$ peak to an analytic model derived considering the emission of a relativistic blast wave expanding into an external medium. The prediction of the model for the $v F_{v}$ peak evolution matches well with the observations. We observe the blast wave transitioning into the deceleration phase. Furthermore, we assume the expansion of the blast wave to be nearly adiabatic, motivated by the low magnetic field deduced from the observations. This allows us to recover within an order of magnitude the flux density at the $v F_{v}$ peak. Under this scenario, we argue that the distinction between prompt and afterglow emission is superfluous as both early-time emission and late-time emission emanate from the same source. While the external shock model is clearly not a universal solution, this analysis opens the possibility that at least some fraction of GRBs can be explained by an external shock origin of their prompt phase.
\end{abstract}

7th Fermi Symposium 2017

15-20 October 2017

Garmisch-Partenkirchen, Germany

${ }^{*}$ Speaker. 